\title{
EXPERIMENTAL STUDY ON TANGENTIAL CIRCLE CHARACTERISTIC IN HIGH RATIO OF LENGTH AND WIDTH, HEXAGONAL ARRANGED UTILITY BURNER
}

\author{
Xinyu Cao, kai Zhao, Liang Yang, Ning Ding, Xianqiao Zhao, Junhu Zhou, \\ Zhenyu Huang, Jianzhong Liu, Kefa Cen

\begin{abstract}
Clean Energy and Environment Engineering Key Lab of Ministry of Education, Zhejiang University, HangZhou,
\end{abstract} \\ P.R.China, 310027
}

\begin{abstract}
In the large utility burner, tangential circle characteristic of multiangular burners is important guideline of burner. Through the experiments on the cold model test, the paper reports the tangential circle characteristic on high ratio of length and width, rectangle furnace and hexagonal arranged burners, and analyses the inner rule quantificationally. Contact circle has rotation, periodicity, time averaging and crumbliness, and jet flow swing has periodicity with the periodicity of tangential circle.
\end{abstract}

Keywords: Cold Model Test, Rectangle Furnace, Hexagonal Arranged, Tangential Circle Characteristic

\section{FOREWORD}

Tangential circle characteristic is the important index of power plant boilers. Through the experiments on the rectangle furnace and hexagonal arranged burners, the paper reports aerodynamics of boiler, which can provide the basis for the design, operation and reconstructoin of large power plant boilers.

At the same time, the paper also reports the regularity cognition of the tangential circle characteristic in the configuration. The aerodynamics experimentation in power plant boilers is based on the model test-bed (simulation proportion 1:10) of the heavy oil-to-coal water slurry reconstruction item in some internal power plant boiler(410t/h).

\section{TEST-BED, METHOD OF EXPERIMENTATION \& CONDITION TO EXPERIMENTATION}

\subsection{Brief Introduction of test-bed}

For the purpose of approaching to reality, the design and calculation of the test-bed is applied in the approximate methods such as magnifying burner, mobile burner and additional hearth-wind. (simulation proportion 1:10)

The size of model hearth is $807^{*} 1199 \mathrm{~mm}$ (length-breadth: 1.486). The diameter of hypothetical tangential circle is $75 \mathrm{~mm}$. The jet flow of burner is counterclockwisely tangential with the hypothetical tangential circle.

The white iron compounding is simulated to the arch superheater and convenient for observation. The hint and burner group schematic representations of test-bed are in the follow 2 figs.

Primary air burner called coaxial jet flow group is made up of primary air with direct current in external ring and center low-swirl wind in inner ring, while the secondary air and ash fire wind are the direct current jet flows.

\subsection{Method of Experimentation}

The velocity field of entrails is measured by three hole probe and heat bulb, while the analyze in flow field is showed through fireworks trace by video camera.

\subsection{Condition to Experimentation \& Explanation}

The parameter of experimentation in four different environment is showed in the follow forms, from 1 to 2 .

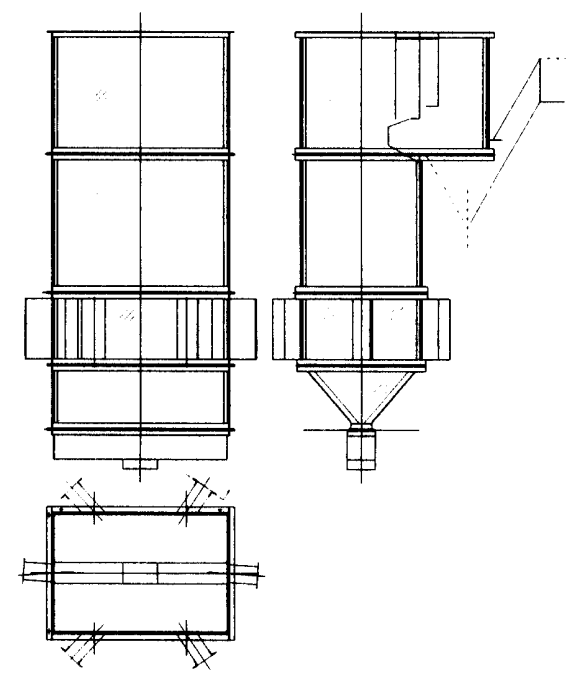

Fig.1 Schematic representation of cold model furnace test-bed 


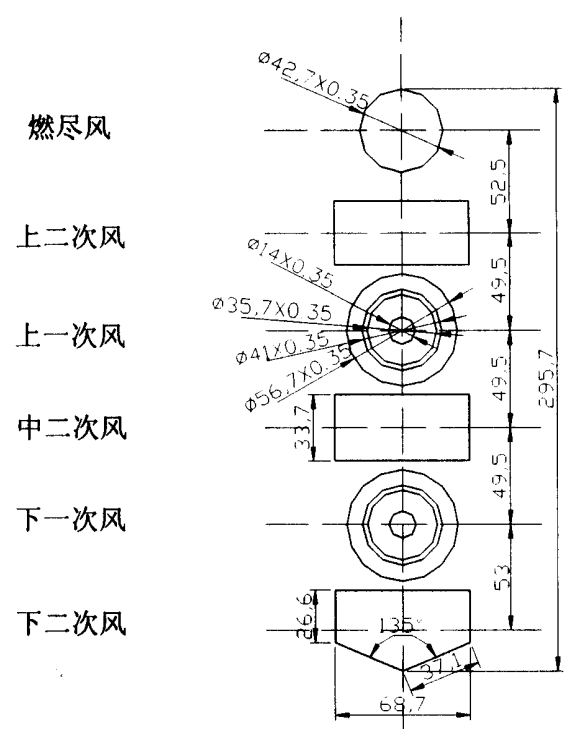

Fig.2 Schematic representation of model burner unit

Tab.1 Declaration of behavior

\begin{tabular}{c|c}
\hline Behavior & $100 \%$ Air(AFW Tangent) \\
\hline Bottom $\operatorname{air}\left(\mathrm{m}^{3} / \mathrm{h}\right)$ & 405.54 \\
\hline
\end{tabular}

Tab.2 Parameter of behavior

\begin{tabular}{c|cccccccc}
\hline Item & AFW & USA & UPA & UMA & MSA & DPA & DMA & DSA \\
\hline Area $\left(\mathrm{mm}^{2}\right)$ & 1385 & 2244 & 1187 & 823 & 2244 & 1187 & 823 & 2264 \\
Velocity $(\mathrm{m} / \mathrm{s})$ & 32.7 & 35.01 & 27.58 & 13.28 & 35.01 & 27.58 & 13.28 & 34.86 \\
\hline
\end{tabular}

Illustration:
AFW- ash fire wind
USA —up-secondary air
UPA — up-primary air
UMA - up-middle air
MSA — middle-secondary air
DPA - down- primary air
DMA - down-middle air
DSA-down-secondary air

\section{CONSEQUENCES OF EXPERIMENTATION \& ANALYZE}

\subsection{The time averaging of tangential circle}

\section{(1) Experimental result}

The fig. 3 shows the cross section of up-primary air tangential circle by three hole probe and heat bulb. The average diameter of tangential circle is $338.7 \mathrm{~mm}$, which approaches to be circularity.

The result of measurement also validates the regulation of that the tangential velocity in the tangential circle' internal reveals to be linear distribution.

(2) Analyze

The measured values by three hole probe and heat bulb is the speed on time averaging. The maximum speed values in different area of the same tangential circle appears to be quasi circularity.

The linear distribution of tangential velocity in tangential circle' internal indicates that the body of rotation composed with tangential circle accords with the regulation of the quasi rigid body circulation.

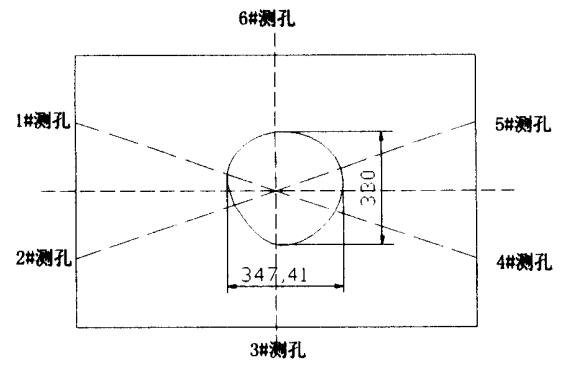

Fig.3 Tangential circle of up-primary air burner level from hot-bulb anemoscope

\subsection{The instantaneity of tangential circle}

(1) Experimental result

The tangential circle in different moments and phase angle is elliptical for an instant, which is analyzed frame by frame by fireworks trace photography.

The tangential circle is elliptical for an instant with $332 \mathrm{~mm}$ major axis and $251 \mathrm{~mm}$ minor axis in length.

(2) Analyze

The reason that the instantaneity of tangential circle appears to be elliptical is due to the impact extrusion effect of two jet flows from left and right.

Because the tangential velocity of tangential circle transforms lineal, the body of rotation composed with tangential circle accords with the regulation of the quasi rigid body circulation. At the same time, the disciform tangential circle don't transform obvious.

The connection between the instantaneity and the time averaging of tangential circle:

The major axis of elliptical tangential circle derived from sectional view by fireworks trace photograph is $332 \mathrm{~mm}$, while the measured value comes from three hole probes and heat bulb is $338.7 \mathrm{~mm}$. So that, the speed on the major axis endpoint of the tangential circle of rotary elliptical quasi rigid body circulation is the maximum. The diameter of the time averaging of tangential circle reflects the major axis length of the instantaneity of tangential circle.

$$
a=2 r
$$

Illustration:

a- - the major axis length of the instantaneity of elliptical tangential circle, $\mathrm{m}$;

$r$ - - the radius of the time averaging of tangential circle, $\mathrm{m}$.

\subsection{The periodicity of tangential circle}

(1) Experimental result

The pictures by frame reveal that elliptical tangential circle rotates on some cycle which is about $0.08 \mathrm{~s}$ (fig.4-14).

(2) Analyze

The reason of periodicity of tangential circle:

On the stable condition, because of the persistent effect of exogenous (jet flow) the periodic circulation of the 
rotary quasi rigid tangential circle is the result of dynamic balance.

For the instantaneity and the time averaging of tangential circle, the periodicity of instantaneous tangential circle can come from the analysze of the time averaging of tangential circle speed:

$$
\begin{aligned}
& T=\frac{2 \pi}{\omega} \\
& \omega=\frac{v}{r}
\end{aligned}
$$

Illustration:

$\mathrm{T}-$ - the cycle of rotary tangential circle

$\omega-$ - the angular velocity of rotary tangential circle

$\mathrm{v}$ - - the linear velocity of rotary tangential circle

$r-$ - the radius of tangential circle (the half length of the major axis of instantaneous elliptical tangential circle)

The linear velocity of time averaging of tangential circle is $13.55 \mathrm{~m} / \mathrm{s}$, while the half length of the major axis is $116 \mathrm{~mm}$, so:

$$
\begin{aligned}
& \omega=\frac{v}{r}=\frac{13.55}{0.116}=81.62(\mathrm{rad} / \mathrm{s}) \\
& T=\frac{2 \pi}{\omega}=\frac{2 \pi}{81.62}=0.077(\mathrm{~s} / \mathrm{r})
\end{aligned}
$$

The frequency of circulation:

$$
f=1 / t=1 / 0.077=12.99(r / s)
$$

The analyze to the cycle of rotary tangential circle frame by frame by photograph also accords with it:

$$
T=\frac{2 \pi \times r}{v}
$$

Illustration:

$\mathrm{T} \longrightarrow$ the period of rotary tangential circle, $\mathrm{s}$;

$\mathrm{v}-$ - the time averaging tangentialvelocity of tangential circle, $\mathrm{m} / \mathrm{s}$;

$r-$ - the radius of the time averaging of tangential circle, $\mathrm{m}$.

By the same way, the angular velocity of rotary tangential circle in the third condition is $63.85 \mathrm{rad} / \mathrm{s}$, which takes up $78.23 \%$ of the first condition, nearly $80 \%$. But the jet flow speed of primary air on the testing section in the first condition is $27.58 \mathrm{~m} / \mathrm{s}$, while the speed in the third condition is $22.55 \mathrm{~m} / \mathrm{s}$.

Obviously, the vortical frequency of tangential circle is proportional to the jet flow speed of primary air on the testing section.

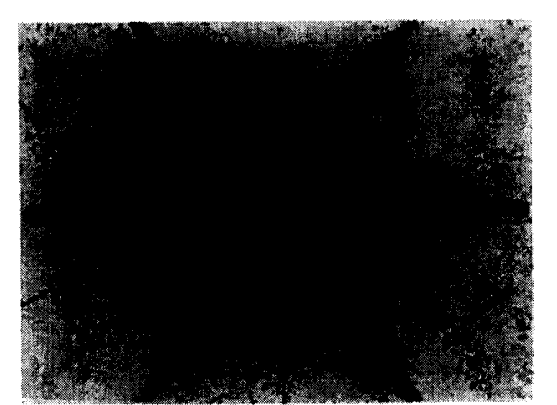

Fig.4 Instant contact circle a from image pick-up

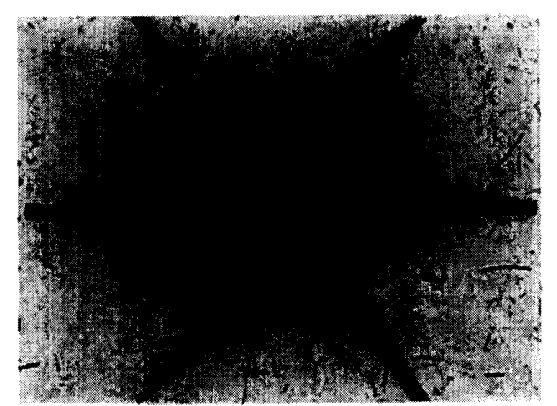

Fig. 5 Instant contact circle $b$ from image pick-up

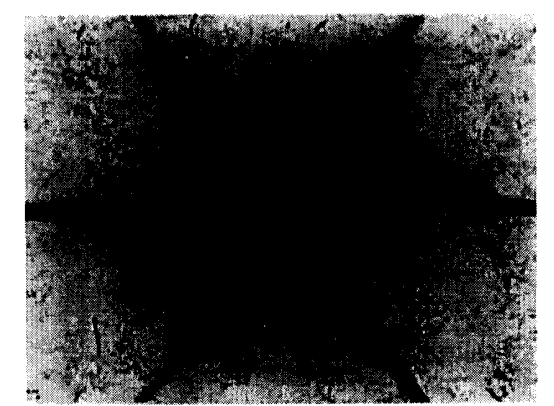

Fig. 6 Instant contact circle c from image pick-up

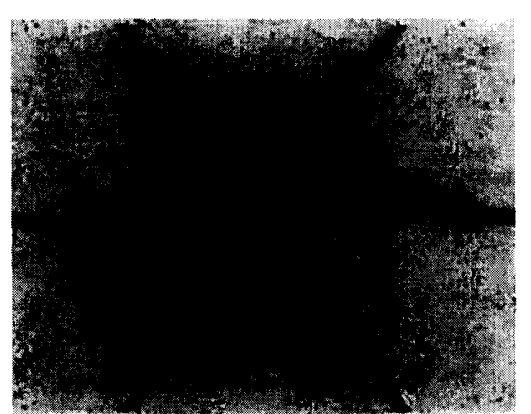

Fig. 7 Instant contact circle $d$ from image pick-up

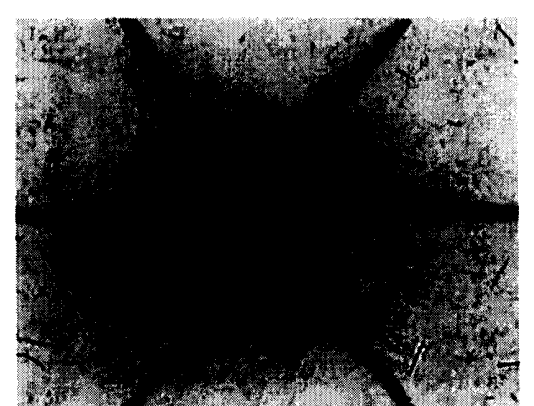

Fig.8 Instant contact circle e from image pick-up 


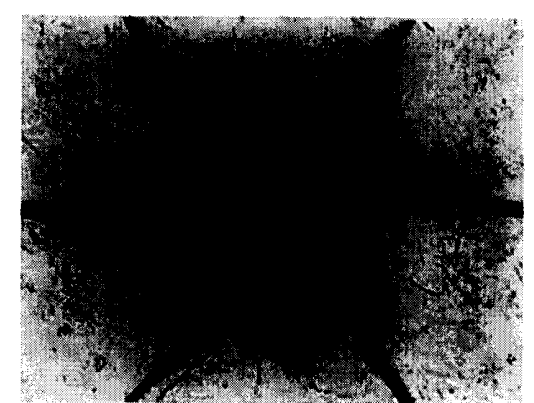

Fig.9 Instant contact circle f from image pick-up

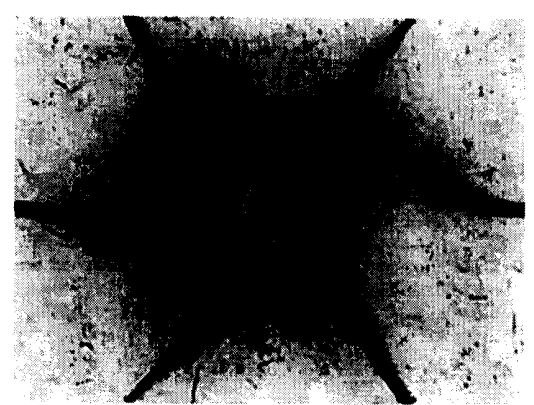

Fig. 10 Instant contact circle $\mathrm{g}$ from image pick-up

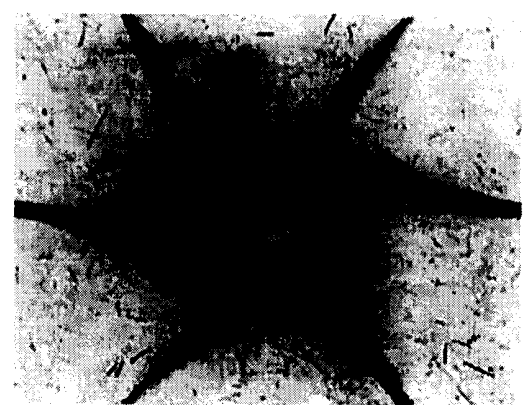

Fig.11 Instant contact circle $h$ from image pick-up

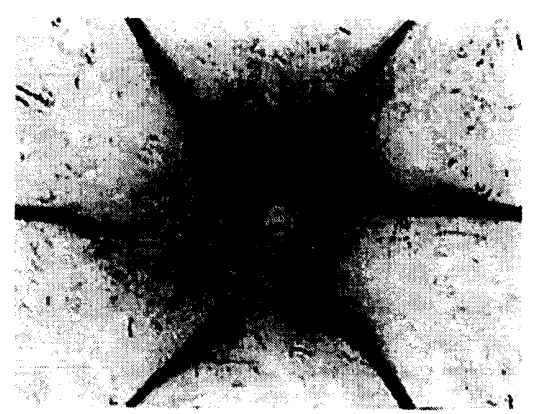

Fig. 12 Instant contact circle i from image pick-up

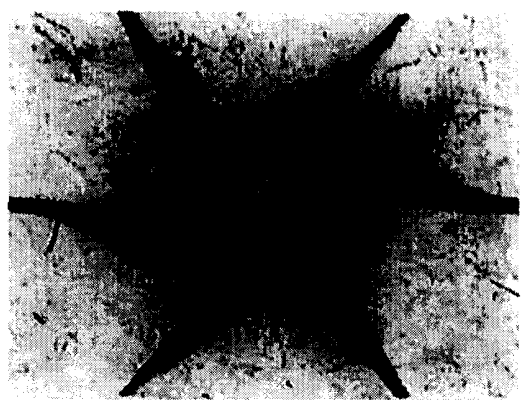

Fig.13 Instant contact circle j from image pick-up

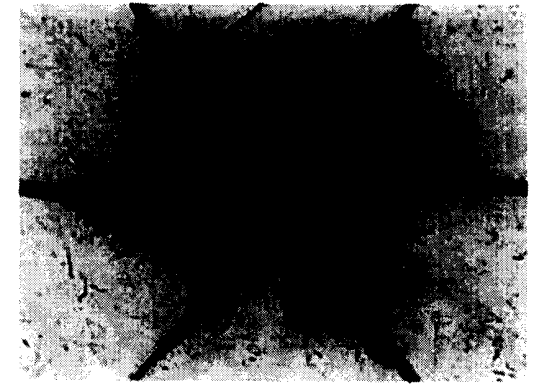

Fig. 14 Instant contact circle $\mathrm{k}$ from image pick-up

\subsection{The crumbliness of tangential circle}

The tangential circle breaks up at some time through the observation to photograph(fig.15). Its form is similarity to be radiating nebula or anomalistic. But the periodicity of it has not been found.

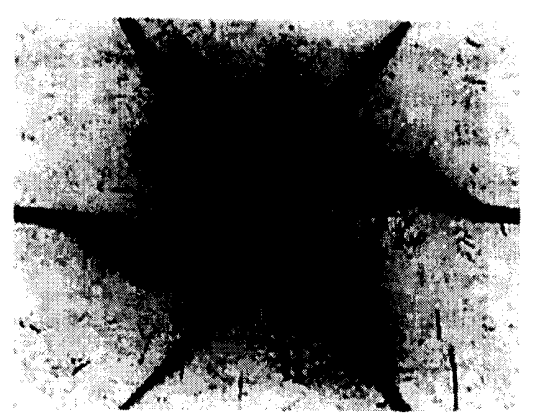

Fig. 15 Instant fragmentation contact circle from image pick-up

The crumbliness of tangential circle is considered to be contact with elliptical tangential circle.

The crumbliness of tangential circle appears when the major axis of rotary elliptical tangential circle is normal to some jet flow. As a result of jet flow pulsation or momentum transformation, the impulse of jet flow is stronger than the bearing power for maintaining the borderline of quasi rigid body tangential circle, what induces to the crumbliness of tangential circle.

The damage of crumbliness:

After the crumbliness of tangential circle, the flame diffuses around, which causes slaggonding and deteriorate the combustion and heat transmission in the combustion space.

Therefore, the air distribution of every jet flow should be stable on the condition of high ratio of length and width hexagonal arranged burner.

\subsection{Cycle wiggle of jet flow and the strengthen to perturbation}

\section{(1) Experimental result}

The jet flow wavers tempestuously before contacting with the tangential circle (Fig.4-14). The waver possesses periodicity.

(2) Analyze

The reason of jet flow waver:

The elliptical tangential circle with recurrent circulation accords with the characteristic of quasi rigid body, whose major axis and minor axis pass by of some burner alternately. The jet flow accomplishes recurrent 
waver when the terminal of major axis skates over some jet flow of one burner immediately.

In this way, the cycle of waver to jet flow is a half on the cycle of rotary tangential circle:

$$
T_{j e t}=\frac{T}{2}
$$

Illustration:

$\mathrm{T}_{\text {jet }}-$ - the cycle of waver to jet flow of burner, $\mathrm{s}$;

$\mathrm{T}$ - - the cycle of rotary elliptic tangential circle, $\mathrm{s}$.

Predictably, for the waver to jet flow recurrently the intensity of turbulence can increase by leaps and bounds, what strengthens the ability of heat transfer in the space of boiler.

\section{CONCLUSIONS}

Through the experimentation of flow field on the cold state modeling test-bed $(1: 10)$ based on the high ratio of length and width hexagonal arranged burner $(410 \mathrm{t} / \mathrm{h})$, the primary conclusion is followed:

The periodical circulation of tangential circle in flow field follows with the regulation of rigid body. The major axis length of the instantaneity of elliptical is equal to the semidiameter of the time averaging tangential circle.

The period of rotation of tangential circle accords with the followed regulation and the rotational frequency of tangential circle is proportional to the burden.

$$
T=\frac{2 \pi \times r}{v}
$$

Illustration:

$\mathrm{T}$ - the period of rotary tangential circle, $\mathrm{s}$;

$\mathrm{V}-\mathrm{t}$ the time averaging tangentialvelocity of tangential circle, $\mathrm{m} / \mathrm{s}$;

$\mathrm{r}^{-}$- the radius of the time averaging of tangential circle, $\mathrm{m}$.

When the perturbation is visible, the elliptical tangential circle may be broken.

The jet flow of some burner wavers recurrently on the horizontal direction whose frequency is as twice as the frequency of revolvable tangential circle. The waver can increase the intensity of turbulence in flow field and strengthen the heat transfer of interspace.

\section{REFERENCES}

[1] Yuxiao Zhao, Bin Sun. Study on Gas-flow Rotation Characteristics in Furnace of Six-corner Tangentially Fired Boiler. NORTH CHUINA ELECTTRIC POWER. No.12,(2001),

[2] Changfu You, Haiying Qi. EXPEMMENTAL INVVESTIGATION ON GAS FLOW IN A MODEL OF TANGENTIALLly-FIRED BOILER.JOURNAL OF ENGINEERING THERMOPHYSICS. Vol.22, No.3,(2001),

[3] Zhijun Zhou, Zhenyu Huang. Jet deflection and analytic calculation of the actual furnace tangential circle. Journal of Zhejiang University (Engineering Science).Vol.34 ,No.6,Nov. (2000).

[4] Ruiyang Li,Yuxiao Zhao.STUDY ON GAS-SOLID TWO FLOW FIELD AT BURNER REGION OF SIX CORNERS TANGENTIALLY FIRED BOILER.Proceedings of the CSEE.Vol.22,No.4Apr. (2002).

[5] Yongjie Tan, Guohua Xin. Research on Cold Test for Gas Temperature Deviation of Boiler with Six Angles Tangential Firing. Jilin Electric Power. (2001.4).

[6] JEMMA N B. Break are duration and contact erosion in automotive application [J].IEEE Trans on CMPT: Part A,(1996),19(1):82-86.

\section{USED SYMBOLS}

a the major axis length of the instantaneity of elliptical tangential circle, $\mathrm{m}$

$f$ the frequency of circulation, $r / s$

$r$ the radius of the time averaging of tangential circle, $m$

$\mathrm{T}$ the period of rotary tangential circle, $\mathrm{s}$

$\mathrm{T}_{\text {jet }}$ the cycle of waver to jet flow of burner, $\mathrm{s}$

$\mathrm{v}$ the linear velocity of rotary tangential circle, $\mathrm{m} / \mathrm{s}$

$\omega$ the angular velocity of rotary tangential circle, $\mathrm{rad} / \mathrm{s}$ 